\title{
ON FRINGE FIELD EFFECTS IN THE FERMILAB 50 GEV MUON STORAGE RING *
}

\begin{tabular}{|c|c|c|c|}
\hline \multicolumn{4}{|c|}{ C. Johnstone ${ }^{\dagger}$, F. Méot ${ }^{\ddagger}$} \\
\hline $\begin{array}{l}\text { Abstract } \\
\text { This paper examines the effects of quadrupole fringe }\end{array}$ & \multicolumn{3}{|c|}{ Table 1: Storage Ring Parameters at 50-GeV } \\
\hline This paper examines the effects of quadrupole fringe & Circumference & $\mathrm{m}$ & 1752.8 \\
\hline fields in the FERMILAB $50 \mathrm{GeV}$ muon storage ring Fea- & Neutrino decay fraction & & $39.2 \%$ \\
\hline ibility I Study. This is done by means of precision multi- & Production region: & & \\
\hline In ray-tracing and shows no important contributions from & matching and dispersion suppression & $\mathrm{m}$ & 44.1 \\
\hline ringe fields in terms of dynamic aperture (as long as harm- & High- $\beta$ FODO straight & $\mathrm{m}$ & 688 \\
\hline ful resonances are avoided), in present disagreement with & $\beta_{x \max } / \beta_{y \max }$ & $\mathrm{m}$ & $435 / 484$ \\
\hline milar studies which are the subject of earlier publication. & $\nu_{x} / \nu y$ & & 13.63/13.31 \\
\hline 1 INTRODUCTION & $\begin{array}{l}\text { natural chromaticity } \\
d \nu_{x} / d \frac{\epsilon_{x}}{\pi} / d \nu_{y} / d \frac{\epsilon_{y}}{\pi} \text { (sextus on) }\end{array}$ & & $\begin{array}{c}-23.9 /-23.9 \\
-3.8 /-11\end{array}$ \\
\hline
\end{tabular}

Unprecedented apertures are required in the muon storage ring for a Neutrino Factory because of the exceptionally large emittances associated with the intense muon beams which must be accepted. In the FNAL 50-GeV storage ring, the superconducting arc quadrupoles require a $16-\mathrm{cm}$ bore which leads to strong, extended fringe fields proportional to the poletip field of the quadrupole $(6 \mathrm{~T}$, in this ring). With the need to minimize arc lengths in order to maximize the percentage of muons which decay in the neutrino production straight, the poletip field and the fringe fields cannot be reduced by correspondingly lengthening the quadrupoles. Hence the impact of quadrupole end fields on the performance of this ring must be carefully evaluated.

The effects of quadrupole fringe fields on various machine parameters and on transverse and momentum acceptance in the FNAL $50 \mathrm{GeV}$ muon storage ring [1] are investigated by means of multiturn ray-tracing [2]. Unless otherwise mentioned, the design parameters, etc., are those used in Ref. [3] in view of further comparisons.

In the following, we first establish the basic optical properties, including 4D+momentum acceptance, of the chromaticity corrected ring in the absence of fringe fields, for reference and possible comparisons with other design codes. Next, fringe fields are introduced in arc+production+return quadrupoles and 4D+momentum tracking is performed with nominal beam emittances. Eventually fringe fields in matching section quadrupoles are discussed.

\section{SECOND ORDER MACHINE}

The muon storage ring for a Neutrino Factory is a simple racetrack geometry consisting of a high-beta straight designed for low-divergence neutrino-beam production (the production straight), an opposing straight (the return

\footnotetext{
* Work supported by the Universities Research Association, Inc., under contract DE-76-CH03000.

${ }^{\dagger}$ FERMILAB, Batavia, IL, USA (johnstone@cns40.fnal.gov)

$\ddagger$ CEA DSM/DAPNIA/SEA, 91191 Saclay, France (fmeot@ cea.fr)
}

straight), and two arcs with their associated dispersion suppression and matching sections. The important physical and optical parameters, in particular those which relate to the quadrupoles, are given in Tables 1, 2.

Table 2: Large-momentum acceptance arc cell parameters.

\begin{tabular}{llc} 
Dipoles: dipole length & $\mathrm{m}$ & 2.4 \\
dipole bend & $\mathrm{rad}$ & 0.0859 \\
dipole field & $\mathrm{T}$ & 6.0 \\
beam size $(6 \sigma$, max $), \mathrm{W} \times \mathrm{H}$ & $\mathrm{cm}$ & $8.0 \times 5.3$ \\
dipole full aperture**, WxH & $\mathrm{cm}$ & $12 \times 9.3$ \\
sagitta & $\mathrm{cm}$ & 2.67 \\
Quadrupoles: length & $\mathrm{m}$ & 1 \\
arc quadrupole strength & $\mathrm{m}^{-2}$ & .31 \\
arc quadrupole poletip field & $\mathrm{T}$ & 3.6 \\
beam size $(6 \sigma), \mathrm{WxH}$ & & \\
F quad & $\mathrm{cm}$ & $9.2 \times 2.6$ \\
D quad & $\mathrm{cm}$ & $4.2 \times 6.2$ \\
arc quadrupole bore** & $\mathrm{cm}$ & 14 \\
Arc FODO cell parameters: & & \\
cell length & $\mathrm{m}$ & 9.8 \\
cell phase advance & $\mathrm{deg}$ & 90 \\
$\beta_{\text {max }}$ & $\mathrm{m}$ & 16.2 \\
$D_{x}($ max $)$ & $\mathrm{m}$ & 1.3 \\
total number arc cells & & 31 \\
\hline
\end{tabular}

**aperture $=$ beam size + liner thickness + beam-stay-clear

We convey here the basis material by first producing and checking main parameters and 4D+momentum acceptance of the chromaticity corrected machine (i.e., no fringe fields and arc sextupoles switched on to the levels specified in Table 2).

As an illustration, in addition to recovering tune and chromaticity values as given in Table 1 , ray-tracing provides the $4 \sigma$ beam envelopes shown in Fig. 1, in excellent agreement with matrix methods.

Large acceptance motion is shown in Fig. 2: 400-turn sample phase space trajectories (observation point is at the 


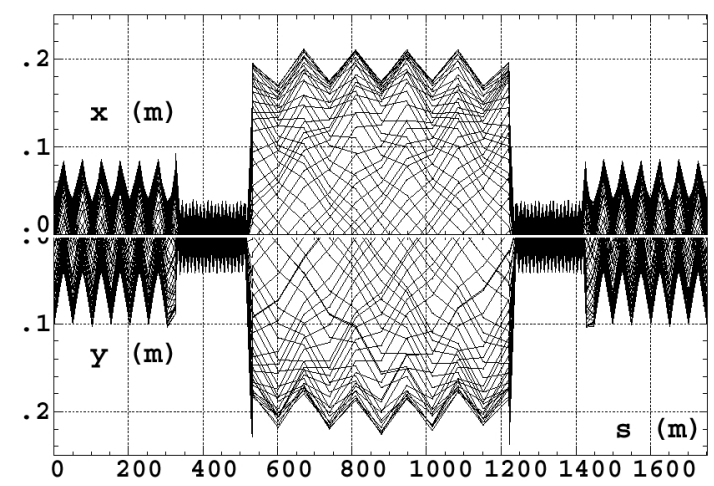

Figure 1: Horizontal (top) and vertical (bottom) $4 \sigma$ beam envelopes, from ray-tracing.
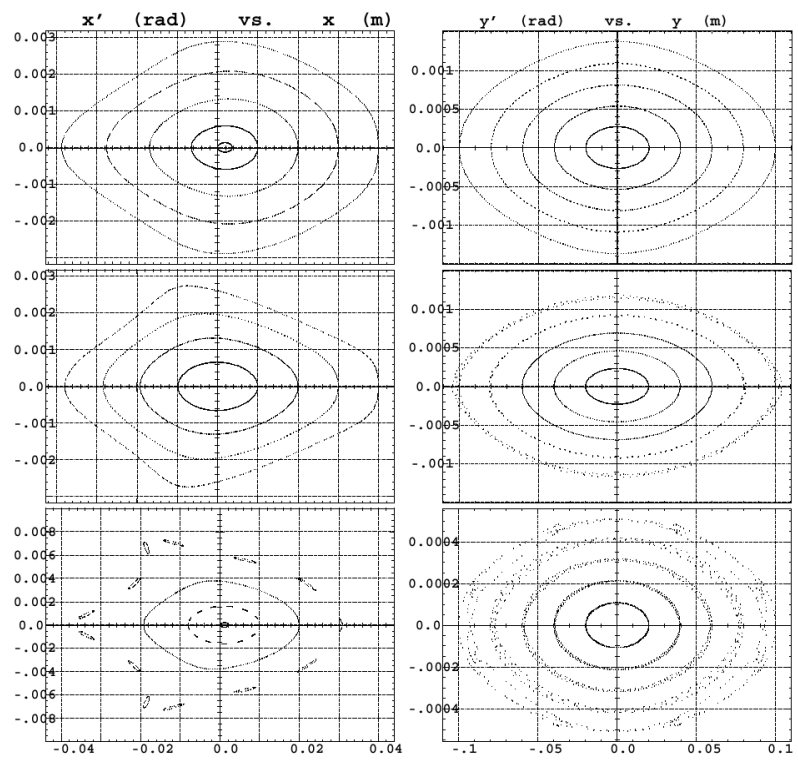

Figure 2: Phase space plots up to DA region, no fringe fields, sextupoles are on. All particles survive except the largest amplitude one in the bottom left-hand plot (with initial conditions $x_{0}=0.04 \mathrm{~m} \approx 4 \sigma_{x}, \delta p / p=-2 \%$ )

center of a defocusing quadrupole in the return straight section) of particles launched with initial coordinates $x_{0}=$ $1-4 \times 10^{-2} \mathrm{~m} \approx 4 \sigma_{x}$ and $\epsilon_{x}=0$ (left column in the figure), or $y_{0}=1-10 \times 10^{-2} \mathrm{~m} \approx 4 \sigma_{y}$ and $\epsilon_{x}=0$ (right col.), and with, top row : $+2 \%$ off-momentum ; middle : on-momentum ; bottom row : $-2 \%$ off-momentum.

A first comparison with tracking results in Ref. [3] is in order. For instance the on-momentum tracking, middlerow plot in Fig. 2 is supposed to compare with Fig. 4 in Ref. [3] (simulations done with COSY), which is not quite the case : the former displays smooth horizontal and vertical phase space motion whereas the latter shows perturbed motion in the $4 \sigma_{x, y}$ range ; similar remarks hold as to comparison with MAD simulations [3, Fig. 5].
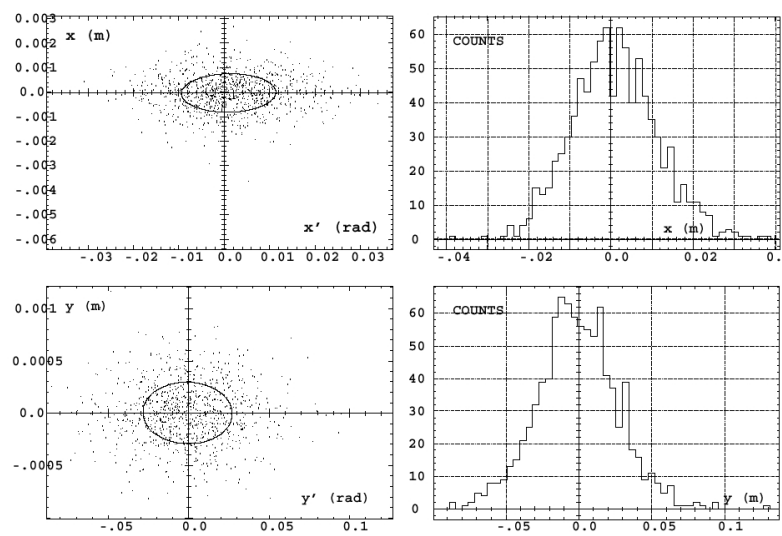

Figure 3: 1000-particle beam ray-tracing, 200 turns. Top row : horizontal motion; bottom row : vertical motion.

As to off-momentum motion the top- and bottom-row plots in Fig. 2 are supposed to compare with Ref. [3, Fig. 14] and [3, Fig. 15] (MAD case) ; again ray-tracing features rather regular phase space trajectories with no acceptance limitation except for the $4 \sigma_{x},-2 \%$ off-momentum particle (bottom-row, left-hand plot in Fig. 2) whereas Ref. [3, Figs. 14, 15] show diffusion or spiral motion, and dynamic aperture far below $4 \sigma_{x}$ in the $-2 \%$ off-momentum case (second plot in Ref. [3, Fig. 14]). Comparison with COSY data [3, Figs. 16, 17] shows strong amplitude limitation within $\pm 1 \%$ momentum range, whereas ray-tracing finds no less than $\approx 3 \sigma_{x}$ limit, in the $-2 \%$ off-momentum case (Fig. 2, bottom, left-hand). Note that, the pronounced spiral behavior in Ref. [3, Figs. 16, 17] makes these data questionable.

These three series of results raise the issue of the validity of one or the other of the simulation data, from the very elementary case of the second order fringe field free machine.

For completion we now launch a 1000-particle beam for 200-turn ray-tracing in the fringe field free, chromaticity corrected storage ring. Particle coordinates $x, x^{\prime}, y, y^{\prime}, \delta l, \delta p / p$ are sorted at random within 2-D Gaussian distributions truncated to $3 \sigma$, yielding in particular the rms sizes $\sigma_{x}=10^{-2} \mathrm{~m}, \sigma_{y}=2.510^{-2} \mathrm{~m}$ corresponding to the nominal emittances $\epsilon_{x}=\epsilon_{y}=$ $7 \pi 10^{-6}$ m.rad whereas momentum spread rms value considered is $\sigma_{\delta p / p}=10^{-2}$. Final phase-spaces and corresponding coordinate histograms are shown in Fig. $3: 921$ particles survive.

\section{FRINGE FIELDS SET}

Fringe fields are now set in arc+production+return quadrupoles (not in matching section ones) and accessorily in the arc sextupoles as well. These quadrupole fringe fields are identical to Ref. [3] ones in order to permit comparisons, with typical shape as shown in Fig. 4 ; they in- 

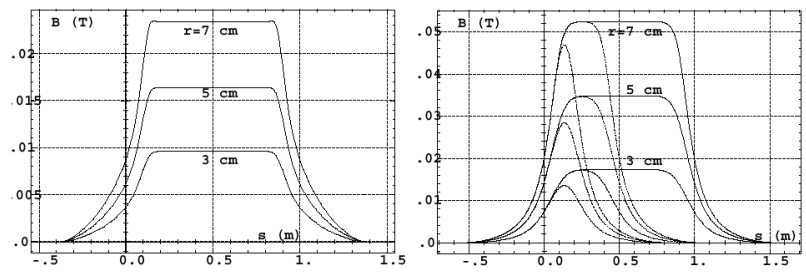

Figure 4: Magnetic field $B(s)$ observed 3, 5 or $7 \times 10^{-2} \mathrm{~m}$ offaxis along the quadrupoles. Left : arc quadrupole (QF1, QD1 families) including sextupole component. Right : $1 \mathrm{~m}, 0.5 \mathrm{~m}$ or $0.27 \mathrm{~m}$ long matching quadrupoles.
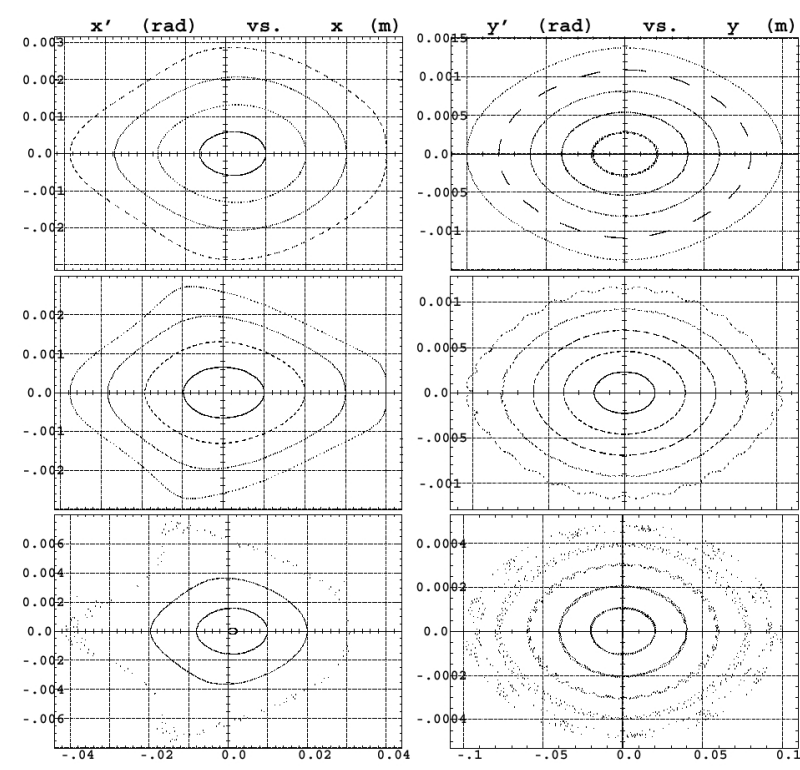

Figure 5: Phase space trajectories, 400-turn ray-tracing, fringe fields set. All vertical motions survive, as well as $+2 \%$ and onmomentum horizontal motion, whereas $-2 \%$ off-momentum horizontal motion shows $\sigma_{x}$ acceptance limitation.

duce sensible perturbation of tunes that are hence first of all re-adjusted to their original, fringe field free values, by means of the sole arc quadrupoles QF1/QD1. Note that, this crude readjustment induces in the production section sensible beta beating which we choose to leave with in the following for simplicity, considering however that it favors our demonstration owing to its inducing larger beam excursions.

Fig. 5 shows phase space plots obtained from 400-turn runs, not very different from the fringe field free case (Fig. 2), from what it can be concluded that their is no evidence of fringe field induced dynamic aperture reduction, at least within the geometrical acceptance and $\pm 2 \%$ momentum bite investigated so far.

Again these result infirm Ref. [3], as observed from com- parison between Fig. 5 and [3, Figs. 18, 19], which is not surprising since similar disagreement already arose in the former discussed fringe field free situation. The spiral behavior however in [3, Figs. 18, 19] again makes these questionable, however they were obtained from 7 th order Taylor maps which may not be sufficient.

Eventually we launch a 1000-particle beam for 200-turn ray-tracing, with random initial beam conditions as before, that yield pahse spaces and histograms similar to those shown in Fig. 3 ; in particular 964 particles over 1000 survive, that is, about the figure of the fringe field free case.

\section{On matching section quadrupoles fringe fields}

In the shorter matching quadrupoles $(0.5 \mathrm{~m}, 0.27 \mathrm{~m}$ or $0.25 \mathrm{~m}$ long ones) the magnetic length is prohibitively shortened by the field fall-offs. This demands an increase of field at pole tip (mostly in quadrupoles QMD1,QFF2,QMF1) so as to restore the appropriate gradient integral $\int G(s) d s$, which is out of the scope of the present study. Apart from that, given the absence of noticeable effects of fringe fields in the production quadrupoles that experience similarly strong beta amplitudes, it is expectable that fringe fields in matching quadrupoles will not change drastically things. Note that, similar question arose as to the harmfulness of the 1 meter long high- $\beta$ matching section quadrupoles in the CERN $\mu \mathrm{SR}$, that were eventually shown to have but negligible influence [4].

\section{CONCLUSION}

The present study on quadrupole fringe fields in the $50 \mathrm{GeV}$ muon storage ring FNAL design, including 4D+momentum tracking, shows no significant effects in terms of dynamic aperture, at least within the $4 \sigma_{x, y}$ geometrical and $\pm 2 \%$ momentum acceptance investigated, in the nominal (MAD data) working tune region $Q_{x}=$ $0.6229, Q_{y}=0.3147$. Observed transverse and momentum acceptance limitations in extreme cases are due mostly to the chromaticity sextupoles.

These results appear to be in sensible disagreement with earlier similar investigations [3].

\section{REFERENCES}

[1] http://www.fnal.gov/pub/hep_descript.html

[2] Zgoubi users' guide, F. Méot and S. Valéro, FNAL Report FERMILAB-TM-2010 (1997) and CEA-Saclay DSM/DAPNIA/SEA-97-13 (Oct. 1997) ; The ray-tracing code Zgoubi, F. Méot, NIM A 427 (1999) 353-356.

[3] Fringe fields and dynamic aperture in the FNAL muon storage ring, F. Zimmermann et als., CERN-SL-2000-011 AP (May 4, 2000).

[4] On the effects of fringe fields in the CERN $50 \mathrm{GeV}$ muon storage ring, F. Méot, report CEA DSM/DAPNIA/SEA-0002, Saclay, April 2000. 